Article

\title{
STREAMLINE'S SHAPE THEORY
}

\author{
Yuvaraj George ㅁo000-0003-4396-1402
}

Chair 101- Aircraft design, Faculty No.1-School Of Aeronautics,Moscow Aviation Institute, Moscow, Russian Federation; georgeyuvaraj2@gmail.com

\begin{abstract}
This article attempts to propose a mathematical model and potential explanation regarding the unavoidable impact of a rigid body's peculiar shape on the seamless flow over it. The solid body completely immersed in a Newtonian fluid and respectively has a relative open circuit flow on it will typically experience various observable phenomena. These typical phenomena in laminar flow are explained using the proposed theory rather than conventional approximations or several partial theories. This article respectively represents an understanding of the laminar flow over a rigid body's external surface with due respect to its distinctive shape and size. To formulate a more realistic and simplified mathematical model for open circuit laminar flow over a body, a mathematical model is proposed based on the historical data of aerodynamics and theoretical mechanics. This mathematical model is intended to properly estimate forces on the continuous surface of the body in a laminar flow, to properly explain, understand and predict various phenomena like flow separation, flow transition, down-wash, stalling at the higher angle of attack, stalling velocity and how cambered airfoil can typically generate lift at a zero incidence angle. Most of all a mathematical model and the mechanism of streamline formation in an open-circuit laminar with respect to the shape and size of the body are illustrated.
\end{abstract}

Keywords: Mathematical Model ; Streamline ;Shape of the body ; Theory

\section{Introduction}

Fluid dynamics remain a peculiar field of study as the fluid particles are very miniature in their dimensions along with negligible masses and the solid bodies interacting with them have a considerable mass and size. Therefore, it is often ambiguous to identify which particular theories among classical mechanics, or quantum mechanics are applicable for a given model of a fluid dynamic system. In the context of Philosophy and History of the fluid dynamics, the approach of classical mechanics has been observed to be not very effective in describing and predicting an open-circuit flow.The popular Navier-Stokes equation is based on Isaac Newton's second law to fluid motion, together with the assumption that the stress in the fluid is the sum of a diffusing viscous term (proportional to the gradient of velocity) and a pressure term hence describing viscous flow. Though Navier-Stokes equation has a wide range of practical applications, it has not yet been proven whether solutions always exist in three dimensions and if they do exist, whether they are smooth i.e. they are infinitely differentiable at all points in the domain. Moreover, Navier- Stokes equations are not conservation equations, but rather a dissipation system, in the sense that they cannot be put into the quasi-linear homogeneous form and Newton's second law is an approximation that is increasingly worse at high speeds because of relativistic effects[11]. Mathematically, Newton's $2^{\text {nd }}$ law can be written as:

$$
\vec{F}=m \frac{d \vec{v}}{d t}=m a
$$

But the above statement seems to disagree with the historical observation by nearly 50 percent and what happened to the other 50 percent of force and how to contemplate that with appropriate assumptions.Consider a dimensional analysis for Newton's second law with the product of dynamic pressure and area. Thus, the product of pressure and area shall be equal to the rate of change of momentum of a body[11].

$$
\vec{F}=\frac{1}{2} \rho \vec{v}^{2} \times A=\frac{1}{2} \frac{m}{l^{3}} \frac{l^{2}}{t^{2}} \times l^{2}=\frac{1}{2} m a
$$


Here, $\vec{F}$ is sum of several forces such as inertial force, gravitational force, capillary force,compressibility or elastic Force, internal force and pressure force. The flow of a fluid in practice does not involve all the forces simultaneously.

Moreover, many theories under circulation increase ambiguity towards the understanding of various phenomena such as flow separation, stalling of the body, transition of flow from laminar to turbulent. All these theories define only a few specific aspects of the flow and each of them tentatively identifies with all the possible effects. Most of all, theoretical literature that can explain few phenomena such as the mechanisms of streamline formation and shock waves formation is observed to be and unavailable.

Fluid dynamics concerning to a rigid body which is relatively moving in a Newtonian fluid more like an aircraft or submarine will undergo various phenomena. Depending on the various factors such as nature of the flowing fluid and nature of the fluid flow the forces acting efficiently on the body can be expressed as functions of fluid density $(\rho)$, dynamic viscosity of the fluid $(\mu)$, free stream velocity $\left(v_{\infty}\right)$, effective $\operatorname{Area}(A)[8]$. But when it comes to the shape like thickness, length, the degree of curvature of the body and its relative effect on the flow, they are often determined by employing the experimental methods[10].

According to Newton's "Philosophiæ Naturalis Principia Mathematica-Book II", the resultant force on a body is a function of the fluid nature, flow nature, shape and size of the body interacting with fluid [6].

$$
\begin{gathered}
\vec{F}=\left\{\rho, \vec{v}, c_{\text {fluid }}, \mu, A, l\right\} \\
\vec{F}=\frac{1}{2} \rho_{\infty} \vec{v}_{\infty}^{2} A_{\text {ref }} C_{F}
\end{gathered}
$$

Equations (3) and (4) gives resultant force on the body which can also be given as a function of similarity parameters like Mach number $(M)$ and Reynold's number(Re)[4][7]. Here, equation (4) gives the resultant force on the body due to the fluid flow over it as a function of flow parameters and area only. Conventionally the resultant force is an aggregate effect of shear stress distribution and dynamic pressure of the fluid over the body[4][7]. But these do not give an explanation why and how the shape is affecting the flow. Often, the vertical component of resultant force and horizontal component of resultant force namely $\operatorname{Lift}(L)$ and $\operatorname{Drag}(D)$ are traditionally written as shown in (5) and (6)[10][7].

$$
\begin{aligned}
& \vec{F}_{y}=\vec{F} \sin (\alpha)=\frac{1}{2} \rho_{\infty} \vec{v}_{\infty}^{2} A_{r e f} C_{L} \\
& \vec{F}_{x}=\vec{F} \cos (\alpha)=\frac{1}{2} \rho_{\infty} \vec{v}_{\infty}^{2} A_{r e f} C_{D}
\end{aligned}
$$

In these equations, the force due to shear stress is ignored, and equations show forces as a product of free stream dynamic pressure $\left(q_{\infty}\right)$ and a reference area $\left(A_{r e f}\right)$. So, there is always a deviation in calculated results with respect to the actual magnitude of the force on the body. Thus, coefficients of forces (dimensionless parameters) and reference area are considered rather than actual parameters. Therefore, there is always a need for experimental methods to evaluate estimated values and to correct them. The corrections obtained from experiments have to be substituted in the equation to obtain near exact calculations[10].

From these, we can undoubtedly understand that not just shape is ignored in extant conventional formulae but the appropriate size of the body is not absolutely considered. The NACA reports regarding camber, chord, and thickness of airfoil is another excellent example to show that not all aspects regarding shape and size are not adequately considered in mathematical models and were evaluated using trail \& error methods. Notably, there is a lack of proper theoretical explanation and a mathematical model regarding how shape affects the continuous flow and so on.

However, solid body interacting with fluid will have negligible effects due to fluid interaction at low speeds. A mathematical model in terms of classical mechanics is more lucid, yet it has its a limitation when continuum effects occur such boundary layers, ionization (mass or energy dissipation) and most importantly classical mechanics studies a system within time intervals. For any continuum effect trajectories 
and forces alone don't play a key role in describing and predicting the system. A fluid dynamic system needs a space-time domain to understand and study the system properly.

Thus, there is a definite necessity for the theoretical fluid dynamics to bridge the gap between classical mechanics and quantum mechanics. Most of all, it is necessary to integrate several partial theories into a single unified theory which can explain these phenomena clearly. At the same time, it shall support a mathematical model that can be useful to predict these phenomenon as-well[3].

In terms of classical mechanics, motion of a fluid particle is studied with due respect to the forces and trajectories[3]. Thus formation of streamline and it's mechanism is vital phenomenon that need to be considered. This will be helpful in order to predict the impact of the shape and size over the flow.

\section{Theory}

\subsection{Fundamental assumptions}

1. The working fluid is typically assumed to be Newtonian fluid.

2. Flow is assumed to be two dimensional laminar flow.

3. Stream-lines are formed due to dynamic pressure and shear stress distribution.

\subsection{Postulates}

1. Fluid particles move freely as their inter-molecular forces are very negligible as long as there is no external influence.

2. When an external solid body typically comes in direct contact with a flowing fluid, the fluid particles bombard at the surface of the body and loose their considerable momentum and kinetic energy[5]. The energy typically lost by a collided fluid particle is convected to surrounding fluid particles as momentum while retaining internal energy.

3. Internal energy is converted to thermal energy and dissipates to the body and flowing fluid in contact with the fluid particles at the point of collision.

4. Similarly, momentum is also transferred to the collided particles from surrounding fluid particles which receive energy to continue the motion of collided particles. So, there will be change in velocity say $\Delta \vec{v}$ and temperature $\Delta T$. This happens as a process of fluid particles trying to achieve thermodynamic equilibrium.Thus, velocity boundary layer and thermal boundary layers are formed, whose characteristics are given by Prandtl's Boundary Layer.[1]

5. However, if the body has enough strength to resist continuous displacement or deformation, the Newtonian fluid will go under displacement or deformation instantly forming a stream line around the body's external surface. Under high speed fluid dynamics shock waves and ionization are observed where internal energy of fluid particles undergo changes forming radicals.

6. The energy lost by flowing fluid on the surface of the body acts as a mechanical load in the form of dynamic pressure normally to body's surface and resistance offered by the surface will be the tangential to fluid flow which results in shear stress distribution[4][7][5].

7. Since the fluid particles are spatially spaced the inter-molecular distance changes and leads to compressibility as they are subjected forces from neighboring fluid particles. Thus, fluids have compressibility under motion.For a given compression (a decrease in volume), the increase in pressure is proportional to the bulk modulus of elasticity E. These effects are continuum.

\section{Conditions}

Now let's assume a rigid body moving in a Newtonian fluid. From above statements the magnitude of the resultant force $|\vec{F}|$ on the body due to relative motion between the fluid and corresponding body can be written as shown in equation (7).

$$
\vec{F}=\left(\frac{1}{2} \rho v^{2}\right) A+\left(\mu \frac{\partial v}{\partial y}\right) A
$$

The resultant force $(\vec{F})$ will have angle with respect to the free stream velocity vector $\left(\vec{\nu}_{\infty}\right)$ or the direction of the free stream. But the induced angle depends not just on angle of attack $(\alpha)$ but also on the shape and 
size of the body. So there exists an angle in between the free stream and resultant of the force which has been commonly observed in asymmetric bodies. This means the resultant force on the body will not be in the direction of the free stream $\left(\vec{F}\right.$ and $v_{\infty}$ are not co-linear). It will have an angle with respect to the free-stream $(\theta)$ even when angle of attack is zero $\left(\alpha=0^{\circ}\right)$. The direction of the fluid particles changed with respect to free-stream forming an angle which resulted in the resultant force to slightly incline at angle $\theta$ with respect to free stream. This change is due to the shape of the body. Thus, the resolution of the resultant force in horizontal and vertical components gives $D$ or $\vec{F}_{X}$ and $L$ or $\vec{F}_{Y}$ equation (8) and equation (9).

$$
\begin{gathered}
\vec{F}_{x}=\left(\frac{1}{2} \rho v^{2} \cos (\alpha+\theta)\right) A+\left(\mu \frac{\partial v}{\partial y} \cos (\alpha+\theta)\right) A \\
\vec{F}_{y}=\left(\frac{1}{2} \rho v^{2} \sin \left(\alpha_{\infty}+\theta\right)\right) A+\left(\mu \frac{\partial v}{\partial y} \sin (\alpha+\theta)\right) A
\end{gathered}
$$

Here, $\theta$ in equations (8) and (9) is the angle between the resultant force due to fluid flow and free stream velocity, due to the change in streamline of particles along its length or simply the shape the of a body[6]. So, as per circulation theory the particles must move around the body in a circle when they fail to circulate they form vortexes.[4] But, in reality fluid particles won't move in a circular path around a body. They will split as two streamline on the upper surface and lower surface of the body. Thus for a circulation around a body $\Sigma \theta$ should be $0^{\circ}$. Which means the resultant force should have no angular deviation with respect free stream velocity. Equations (10) and (11) shows integral form of this circulation condition. Here, in equations (10) and (11) $\theta_{u o}$ and $\theta_{\text {lo }}$ are the deviation of resultant force at the particular point on upper surface and lower surface of the flow respectively.

$$
\begin{aligned}
& \oint_{L E}^{T E} \theta_{u o}=\oint_{L E}^{T E} \theta_{l o}=0 \\
& \oint_{L E}^{T E} \theta_{u o}+\oint_{L E}^{T E} \theta_{l o}=0
\end{aligned}
$$

\section{Calculations}

So let's evaluate the above conditions. To study the impact of shape and size over fluid's flow, let's consider two successive space domains $A_{i} \& A_{i+1}$ in the flow as shown in Figure $1[3]$.

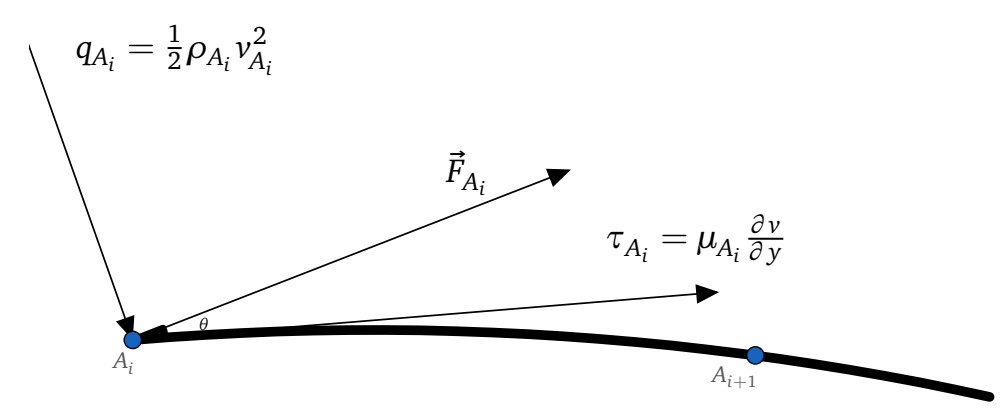

Figure 1. Illustration of the resultant force vector on successive space domains in a stream line

1. Consider a infinitesimally small area as shown in Figure 1 . Figure 1 illustrates that the the force vectors due to dynamic pressure and shear stress of any point $A_{i}$ in streamline which will interact with adjacent points $A_{i+1}$. On any given point $A_{i}$ in the considered system where it has velocity $\vec{v}_{i}$. Upon interaction with other corresponding particles in the streamline, the velocity will change to $\partial \vec{v}_{i}+\vec{v}_{i}$.

2. Thus there will be change in pressure normal to $A_{i}$ which is $\partial q_{A_{i}}+q_{A_{i}}$. The force at $A_{i}$ is normal vector to area. But the corresponding Area $A_{i+1}$ will have an additional force vector on it with certain angle $\theta_{i}$. As per the equations (8), (9) (10) and (11), the force vector is an integral function over the stream lines. The resultant vector will be the sum of all the force vector which is ahead of the 
considered infinitesimally small area $A_{i}$. For 2D flow it can be written as surface integral[8] So, $\vec{F}_{A_{i q}}$ can be written as the resultant fluid dynamic force at a infinitesimally small area due to the dynamic pressure of subjected motion of fluid at $A_{i} . \vec{F}_{A_{i q}}$ is given quantitatively in equation (12).

$$
\vec{F}_{A_{i q}}=\oiint_{A}\left(\frac{1}{2} \rho_{A_{i}} v_{A_{i}}^{2}+\frac{1}{2} \rho_{A_{i q-1}} v_{A_{i-1}}^{2} \cos \left(\theta_{r x y}\right)\right) d A
$$

Here $d A$ is infinitesimally small area between the $A_{i}$ and successive points. $\theta_{\text {rxy }}$ is the algebraic sum of angles between resultant forces vectors $\vec{F}_{A_{i}}$ and $\vec{F}_{A_{i+1}}$ along the streamline over the surface.

3. Similarly, the effect of shear stress distribution of resultant force at $A_{i}$ can be given as:

$$
\vec{F}_{A_{i \tau}}=\oiint_{A}\left(\mu_{A_{i}} \frac{\partial v_{i}}{\partial y}+\mu_{A_{i-1}} \frac{\partial v_{i-1}}{\partial y} \cos \left(\theta_{r x y}\right)\right) d A
$$

Here, Equations (13) gives the effect of shear stress distribution over surface integral. As the second components in the equations (12) to (13) are resultants of the succeeding elements and their effect is dependent on the angle $\theta_{r x y}$.

4. Therefore, the resultant force at a infinitesimally small area $A_{i}$ can be written as sum equations (12) and (13).

$$
\vec{F}_{A_{i}}=\oiint_{A}\left(\frac{1}{2} \rho_{A_{i}} v_{A_{i}}^{2}+\frac{1}{2} \rho_{A_{i-1}} v_{A_{i-1}}^{2} \cos \left(\theta_{r x y}\right)+\mu_{A_{i}} \frac{\partial v_{A_{i}}}{\partial y}+\mu_{A_{i-1}} \frac{\partial v_{A_{i-1}}}{\partial y} \cos \left(\theta_{r x y}\right)\right) d A
$$

Equation (14) gives the resultant force at any given point $A_{i}$, which is the sum of the products of the area with shear stress and dynamic pressure[6][8][3]. The resultant force of the preceding fluid particles can be resolved on the given point with an angle $\theta_{r}$ and the resultant will have an effect on succeeding $A_{i+1}$.

Thus, equation (14) can be simplified as :

$$
\begin{gathered}
\vec{F}_{A_{i}}=\oiint_{A}\left(\frac{1}{2} \rho_{A_{i}} v_{A_{i}}^{2}+\mu_{A_{i}} \frac{\partial v_{A_{i}}}{\partial y}+\vec{F}_{A_{i-1}} \cos \left(\theta_{r x y}\right)\right) d A \\
\vec{F}_{A_{i}}=\oiint_{A}\left(\vec{F}_{A_{i}}+\vec{F}_{A_{i-1}} \cos \left(\theta_{r x y}\right)\right) d A
\end{gathered}
$$

The effect of $\vec{F}_{A_{i}}$ can be resolved in X-plane, Y-plane and Z-plane using trigonometric relations as shown in equations (17), (18) and (19). Here, $\theta_{r_{x}}, \theta_{r_{y}}$ and $\theta_{r_{z}}$ are the angles at which force at any point $A_{i}$ acts on surrounding fluid particles along X-axis, Y-axis and Z-axis respectively.

$$
\begin{gathered}
\Sigma_{0}^{n}\left(\theta_{r_{x}}\right)=\Sigma_{o}^{n} \sin ^{-1} \frac{\sqrt{\left|\vec{F}_{A_{i_{x}}}\right|^{2}-\left|\overrightarrow{V_{i} x}\right|}}{\left|\overrightarrow{V_{i x}}\right|}=0 \\
\Sigma_{0}^{n}\left(\theta_{r_{y}}\right)=\Sigma_{o}^{n} \cos ^{-1} \frac{\left|\overrightarrow{V_{i y}}\right|}{\left|\vec{F}_{A_{i y}}\right|}=0 \\
\Sigma_{0}^{n}\left(\theta_{r_{z}}\right)=\Sigma_{o}^{n} \sin ^{-1} \frac{\sqrt{\left|\vec{F}_{A_{i_{z}}}\right|^{2}-\left|\vec{V}_{i}\right|} \mid}{\left|\vec{V}_{i z}\right|}=\Sigma_{o}^{n} \cos ^{-1} \frac{\left|\vec{V}_{i z}\right|}{\left|\vec{F}_{A_{i z}}\right|}=0
\end{gathered}
$$

Depending upon the direction of flow and it's magnitude the components of $\theta$ can be represented as sin, cos components in the OX,OY planes. Here, $\vec{F}_{A_{i_{x}}}$ and $\vec{F}_{A_{i y}}$ are resolved components of the resultant force in X-plane, Y-plane respectively. where $\vec{V}_{i x}$ and $\vec{V}_{i y}$ are velocity vectors of stream lines in X-plane and Y-plane respectively.

Equations (17),(18) and (19) shows the trigonometric relations between local velocity vector, $\theta$ and force. When the circulation conditions mentioned in equations (10) and (11) are applied, the algebraic sum of $\theta$ 
along the stream line should be zero. Otherwise, streamline formation is disturbed leading to occurrence of other phenomena such as vortex formation at trailing edge or down-wash.

So based on the above equations it can be explained that the formation of stream changes with the degree of curvature of the body, its size, flow velocity, fluid density and dynamic viscosity of the fluid. As per $3^{r} d$ and $4^{t} h$ postulates the energy is transferred or transformed between two time intervals i.e. energy or momentum lost by collided fluid particles and energy received by collided fluid particles from its surrounding fluid particles. So there can be two time intervals $t_{1}-t_{2}$ and $t_{2}-t_{3}$.

At a local area $A_{i}$ the equation (16) can be written as:

$$
\begin{gathered}
\vec{F}_{A_{i}}=\int_{t_{1}}^{t_{3}}\left(\oiint_{A}\left(\vec{F}_{A_{i}}+\vec{F}_{A_{i-1}} \cos \left(\theta_{r x y}\right)\right) d A\right) d t \\
\vec{F}_{A_{i}}=\int_{t_{1}}^{t_{2}}\left(\oiint_{A}\left(\vec{F}_{A_{i}}+\vec{F}_{A_{i-1}} \cos \left(\theta_{r x y}\right)\right) d A\right) d t+\int_{t_{2}}^{t_{3}}\left(\oiint_{A}\left(\vec{F}_{A_{i}}+\vec{F}_{A_{i-1}} \cos \left(\theta_{r x y}\right)\right) d A\right) d t
\end{gathered}
$$

Equation (20) and (21) shows time integral of force at a local area (A) over the two successive events. $t_{1}-t_{2}$ is the time interval when fluid particle collide at the solid body's surface and loose their momentum and time interval $t_{2}-t_{3}$ is the period when collided particles receive momentum to continue their motion. This is due to elasticity of the fluid and its tendency to attain thermodynamic equilibrium. Equation (20) has both time integral and surface integral defining the force and angle at which the it is acting on the corresponding fluid particle. The time integral defines the consecutive effects of the fluid particles motion and surface integral corresponds to local changes on the surface. Where as the $\theta$ corresponds to the degree of the curvature of the body which can be noted by the trigonometric relations shown in equations (17), (18) and (19).

\section{Discussions}

\subsection{Flow over a linear body}

Consider a flat plate parallel to the free-stream with a smooth laminar flow as shown in Figure 2. In the Figure 2 the flat plate 'f' has a viscous laminar flow which is indicated by dotted stream lines. The thickness and style of lines indicate viscosity at that particular point. The free body diagram of ' $f$ ' shows force vectors on upper surface and lower surface from leading edge to trailing edge. Here, $\vec{F}_{u o}$ is the resultant force vector on upper surface and $\vec{F}_{l o}$ is the resultant force vector on lower surface at leading edge. $\vec{F}_{i u}$ and $\vec{F}_{i l}$ are the resultant force at any given point in between leading edges and trailing edges respectively. $\theta_{n}$ is the algebraic sum of all the respective angles of $\vec{F}_{A_{i}}$ over the flat plate i.e $\theta_{n u}+\theta_{n l}=\theta_{n}$. Here, $\Sigma_{0 u}^{n u} \theta=\theta_{n u}$ and $\Sigma_{0 l}^{n l} \theta=\theta_{n l}$.

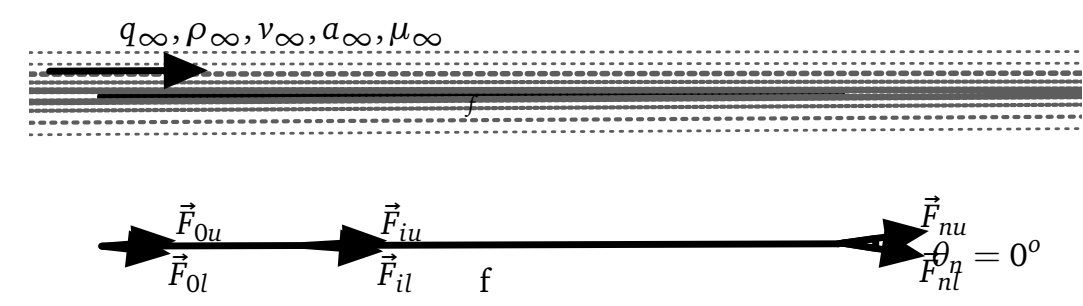

Figure 2. Laminar flow over a flat-plate

So, at stagnation point if the body with stands these forces then substantial phenomenon are observed: Thus, the resultant force of the stagnant fluid particles will be at an angle with respect to free-stream velocity. The resulting force from stagnation point, which is acting over the surrounding fluid particles makes them to flow around the upper surface and lower surface of the body will have change in their $\theta_{i} u$ and $\theta_{i} l$ and magnitude $\vec{F}_{i u}$ and $\vec{F}_{i l}$. As the change in momentum simultaneously effects the viscosity and thus change 
in the velocity vectors of fluid particles moving on the upper surface and lower surface will continuously have a increment in the angle $\theta_{i}$ and the magnitude of the resultant force $\left|\vec{F}_{i}\right|$ till the trailing edge. If the sum of $\theta_{n u} \& \theta_{n l}$ is equal to $0^{\circ}$.[6] Then fluid particles flow in same direction as free-stream as long as the magnitude of $\vec{F}_{n}$ is not too high to deviate them from channeled stream line. Thus at low speed where the magnitude of resultant aerodynamic force at trailing edge $R_{n}$ is not too high and upper and lower surface from stagnation point have same length and symmetric shape the flow remains laminar and smooth. As this is a straight body, the fluid particles will have tangents for free-stream at the leading edges and trailing edges of the body and change in $\theta$ is very minimal. Thus stream lines formed around the flat plate are nearly parallel to it's surface.So, from Figure 2 it can be stated that for a straight line body which is parallel to the free-stream velocity vector $\left(\overrightarrow{v_{\infty}}\right)$ there won't be much change in $\theta_{i}$ as long as the velocity is not too high to disrupt the streamlines formed.

But as the velocity and length of body increases, we observe that the flow separation and turbulence phenomenon posterior to the trailing edge. Even for a thick plate with considerable frontal area under stagnation, we observe this phenomenon at low speeds. Because at higher velocity of the free stream and large length of body, more energy is lost at stagnation point and subsequent fluid particles gain energy and becomes turbulent as the resultant forces increases continuously and thus flow separation occurs as the magnitude of the force at that particular point of separation is too high $\left|\vec{F}_{i}\right|$ to detach the fluid from streamline. Thus fluid particles detach causing irreversible loss of the flow (turbulence) from the stream line if they have enough energy to deviate from their respective streamlines as shown in Figure 3[3].

Similar phenomenon can be observed in flat plates with angle of attack. As more area is opposing the motion of the fluid particles at the area of stagnation is higher and the induced angle of attack contributes to change in the direction of fluid particles as the force is already acting at an angle.[8]Like wise higher velocity of free-stream tends to increase magnitude of resultant force closer to stagnation point. This leads to stall velocity and stalling angle.

Based on Reynold's number we can say that the dynamic viscosity $(\mu)$ is inversely proportional to the turbulence on the surface of the body[8][5][2]. The distance from stagnation point $(l)$, $\operatorname{velocity}(\vec{v})$ and number of fluids particles available to receive momentum and transfer $(\rho)$ are directly proportional to turbulence on the body's surface. [9] Therefore, based on historical data we can say that as long as $\operatorname{Re}<2300$

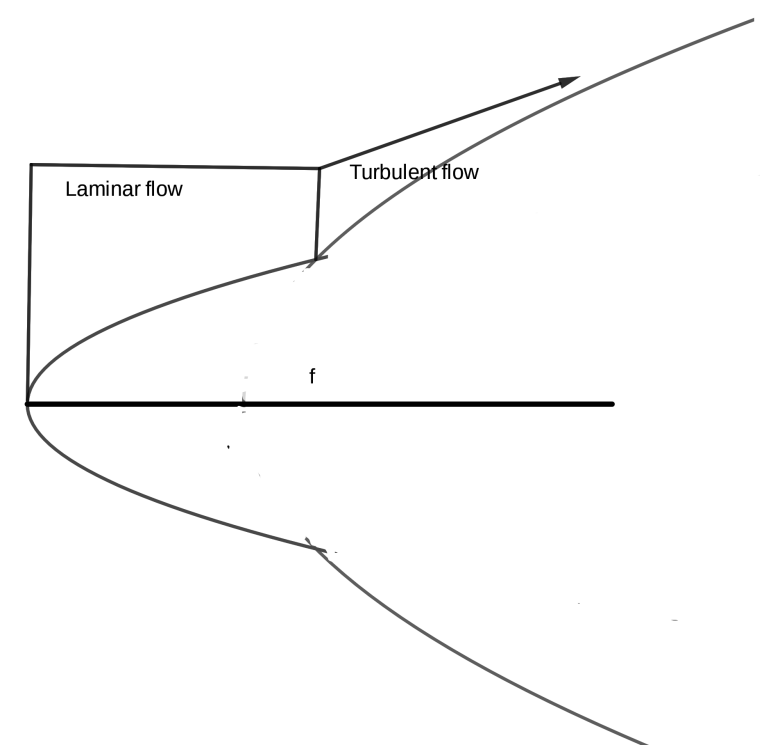

Figure 3. Flow on a flat plate showing boundary layers of laminar and turbulent flows

at any point on the streamline of the flow will be laminar. Here, $l$ in the equation (6.1) must be understood as the length of stream line rather the length of the body.[9] 


$$
R e=\frac{\rho v l}{\mu}\left\{\begin{aligned}
R e<2,300 & \text { laminarflow } \\
2,300<R e<4,000 & \text { transitionalflow } \\
R e>4,000 & \text { turbulentflow }
\end{aligned}\right.
$$

Based on the conditions specified on can evaluate the nature of flow at any given point on the stream. Here, $l$ should be considered from point of stagnation to the point of consideration on the streamline even if it is posterior to the trailing edge. As it is already well known that the transfer of momentum is a continuum effect in the flow. So, the fluid particles will retain the momentum even after passing away from the body and thus they will tend to flow in turbulent nature posterior to the trailing edge.

Similarly a square or rectangular body will undergo above phenomenon and these can be analyzed using fromulae and assumptions mentioned above. But it will have a larger area subjected to stagnation and which will result in larger magnitude of resultant force $|\vec{F}|$. Thus the fluid particles closer to stagnation point will have higher energy. This will contribute to earlier flow transition and flow separation compared to a flat plate or a streamlined body like an airfoil.

\subsection{Flow over a curvilinear body}

Consider a body with circular cross-section as shown in Figure 4. It will have a tangents all around the body, unlike flat plate. So fluid particles will have continuous change in $\theta$ along their streamline. This is due to the change in the body shape of the body. However as the body is symmetric, the magnitude of the resultant force on the upper surface and magnitude of the resultant force on the lower will be equal i.e. $\left|\vec{F}_{i u}\right|=\left|\vec{F}_{i l}\right| .[6][8]$ Similarly, $\theta_{i u}=\theta_{i l}$. As discussed in equation (10) and (11), the line integral of angle $\theta$ around a curved, symmetric body will be zero. So, under low Reynold's number the flow will not experience any separation and turbulence. However, the change in $\theta$ is also continuum depending on the shape of the body[2]. If the change is too significant and sufficient enough for the fluid particles to skip from their stream line with considerable magnitude in the resultant force at a certain point, then flow separation will occur. In this way a symmetric flow separation is occurred when the line of symmetry and free stream vectors are co-linear. This is because the fluid particles will have opposing forces due to the path they traveled and magnitude of the resultant force at the trailing will be high.[6] As fluid particles of same mass and velocity collide which each other they will cause turbulence.

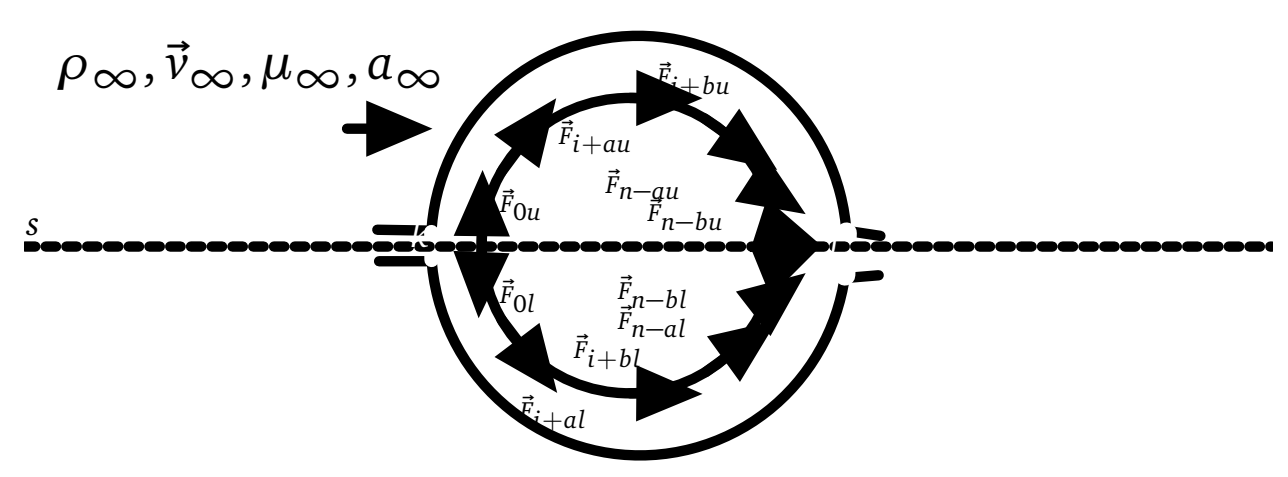

Figure 4. Flow over a circular cross-section body

Now consider a symmetric airfoil which has it's line of symmetric or chord at zero incidence with the free stream. It will not have any vertical force components or simply lift co-efficient is zero $\left(C_{L}=0\right)$. But it will have a coefficient of drag or horizontal force component $C_{D 0}$. This is because the vector of resultant force and its direction is in the same direction as the free-stream. Many phenomena like flow separation, stalling, generation of lift and drag can be explained similar to that of flow over a flat plate and curved body. 
But when the angle of attack is increased, more area is subjected to stagnation compared to flat plate and curved body will have smooth change in $\theta$. Thus symmetric airfoils will have higher performance compared to flat plate at an incidence angle.

Flow over an asymmetric body such as cambered airfoils can be explained in the following way. The asymmetric body will have unequal areas on its upper surface and lower causing flow on both surfaces to have different $\vec{F}_{i}$ and $\theta_{i}$. Where force on one side is greater than other side and the angle $\theta$ on one side of the body is greater than the other side, the resultant forces will be have more magnitude towards one plane even at zero incidence. Thus, camber airfoils will have a lift coefficient $\left(C_{l}>0\right)$ even when $\alpha=0$. However, they will have an effect called down wash. In cambered airfoils the line integrals of $\theta$ on both upper surface and lower surface are not equal. As the length or area of the upper surface is higher than lower surface with higher degree of curvature, It will have $\theta_{u}$, which is greater than $\theta_{l}$. Thus stream lines at trailing edge seems little offset with free-stream. However, this effect is negligible, and the angle of down-wash is nearly $\sim 3^{\circ}$ to $5^{\circ}$ (based on historical data of aerfoils).

\section{Conclusion}

We enough know that the specified thickness of the body effects by progressively increasing Reynold's number due to more stagnation and energizing the fluid particles.[8] The length of the body will equally contribute by contributing to the change in the magnitude of the resultant force at each consecutive point.[6] These can be expressed using equations given in this article. Curvature of the body effects the streamline by contributing to the change in the angle $\theta$ of resultant force at consecutive points from leading edge to the trailing edge. Thus this article mathematically confirms these facts and explains the phenomena such as stalling and effects of camber. With the above mathematical model formation of streamline, flow separation and flow transition can be explained for a given body's shape, size and flow parameters for a two dimensional flow.

With FEM this mathematical model can help to estimate flow over a body i.e. to predict the formation of streamline and changes along the streamline with respect to the free-stream velocity, dynamic viscosity and body's shape and size. This model can also be used to reduce the iterations in a design process especially while performing itinerary shaping and sizing of a body in fluid dynamic system or components with open circuit flow. In designing hydrofoils and airfoils, this model can have a significant impact in terms of shaping and sizing compared to conventional methods. Conventional methods involve itinerary designs which start with arbitrary an or experimental data. In designing a streamlined body the mathematical model presented here presents a lucid and quantitative parameters to design.

Future scope of work involves developing a CFD(computational fluid dynamic) solver based on the mathematical model prominently mentioned in this article. To objectively evaluate CFD solver and mathematical model using experimental methods.

When we implement principles of classical mechanics, the mathematical model is unrealistic and estimated values will have a significant deviation with respect to the real-time phenomenon. Though solid body's shape and size remain constant over the large duration, fluid particles from being static to ionization (under high speeds) will go under various changes that even defies many laws in classical mechanics. Moreover, it is required to extent the current model for a three dimensional flow which can be accommodate not just a laminar flow. A further investigation is required how well this model is applicable for non-Newtonian fluids.

Funding: This research received no external funding

Acknowledgments: I thank Prof. Veeranjaneylu K, MLR Institute of Technology for improvising mathematical model to be more realistic by suggesting proper assumptions, and Mr.Pavan Kumar B, Asst. Design Engg., AST Aersystems Pvt. Ltd. for comments that greatly improved the manuscript quality and readability. I am immensely grateful for both of them for their comments on earlier versions of the manuscript, although any errors in this article are my own and should not tarnish the reputations of these persons.

Conflicts of Interest: "The authors declare no conflict of interest." 


\section{Nomenclature}

$\alpha \quad$ Angle of incidence

$\Delta \vec{v} \quad$ Change in velocity vector of the fluid particles

$\Delta T \quad$ Change in the Temperature

$\mu \quad$ Dynamic viscosity of the fluid

$\rho \quad$ Denisty of the fluid

$\theta \quad \vec{F} \angle v_{\infty}$-angle between the resultant force due to fluid flow and free stream velocity

$\theta_{r_{x}} \quad$ Angle of resolved force component along $\mathrm{X}$-axis

$\theta_{r_{y}} \quad$ Angle of resolved force component along Y-axis

$\theta_{r_{z}} \quad$ Angle of resolved force component along Z-axis

$\theta_{\text {rxy }} \quad$ Angle between two succesive points or space domains along the streamline
$\vec{F}_{x} \quad$ Horozontal component of Force Vertical component of Force Resultant force due to the fluid motion Velocity of the fluid Reference Area Co-efficient of Drag Co-efficient of lift Co-efficent of force length Effective area Acceleration of the fluid Mass of the fluid time interval

\section{Subscript}

$\infty \quad$ Parameters of free-stream velocity

LE Leading Edge

TE Trailing Edge

$A_{i} \quad$ Local parameters such as $\rho, \mu, \vec{F}$ and $\vec{v}$ the area (A)

$A_{i_{q}} \quad$ Local dynamic pressure on the area (A)

$i \quad$ Local parameters or derivatives on the streamline

ix Components of local parameters or derivatives on the streamline in the $\mathrm{x}$-direction or parallel to free-stream

$i u, O u, n u \quad$ Components of local parameters or derivatives on the upper surface of the body

il,Oul,nl Components of local parameters or derivatives on the lower surface of the body

lo Lower surface of the body

uo Upper surface of the body

$t_{1}-t_{2} \quad$ Time period between collision and momentum lost

$t_{2}-t_{3} \quad$ Time between fluid particles stagnation and movement

\section{References}

1. Fifty years of boundary-layer theory and experiment. National Advisory Committee for Aeronautics; Washington, DC, United States https://ntrs.nasa.gov/archive/nasa/casi.ntrs.nasa.gov/20150019333.pdf. Mar 18, 1955.

2. For McGraw-Hill Concise Encyclopedia of Physics:. center of pressure. (n.d.) mcgraw-hill concise encyclopedia of physics. https://encyclopedia2.thefreedictionary.com/center+of+pressure, 2002. [Online; Retrieved March 2 2019 ].

3. Texas A and M University. Fluid mechanics - lecture notes - chapters 1-14,academic year 2014-15. Fluid Mechanics-Lecture notes https://www.studocu.com/en-au/document/texas-am-university/fluid-mechanics/ lecture-notes/fluid-mechanics-lecture-notes-chapters-1-14/422911/view.

4. L J Clancy. Aerodynamics. 1975.

5. Mohamad Hafiz Ghani, Sarah , P X Khaleeda, C W Lim, and Phoa . Design and development of air flow sensor, drag and lift force sensor and pressure sensor for air flow bench part 3. DESIGN AND DEVELOPMENT OF AIR FLOW SENSOR, DRAG AND LIFT FORCE SENSOR AND PRESSURE SENSOR FOR AIR FLOW BENCH, 012016.

6. L M. HOSKINS. Theoretical mechanics; an elementary text-book, 1900.

7. John D Anderson Jr. Fundamentals of Aerodynamics. 2014.

8. G. Biswas S. K. Som. Fluid mechanics nptel(lecture notes on introductory fluid mechanics course). NPTEL-IIT Kharagpur \& IIT Kanpur https://archive.org/details/FluidMechanicsNPTEL/page/n9. Accessed: 2009.

9. Arnold Sommerfeld. "ein beitrag zur hydrodynamischen erkläerung der turbulenten flüssigkeitsbewegüngen (a contribution to hydrodynamic explanation of turbulent fluid motions)". International Congress of Mathematicians, 1908. Pgs:116-124.

10. Andrea Ianiro Stefano Discetti. Experimental Aerodynamics. 03-2017.

11. Wikipedia contributors. Newton's laws of motion - Wikipedia, the free encyclopedia. https://en.wikipedia.org/ w/index.php?title=Newton\%27s_laws_of_motion\&oldid=886801669, 2019. [Online; accessed 2-April-2019]. 